\title{
The Interpersonal Dimension of Online Patient Forums: How Patients Manage Informational and Relational Aspects in Response to Posted Questions
}

\begin{abstract}
The internet has revolutionised the ways in which patients acquire medical information, a development which has clearly been welcomed by patients: seeking out health information online is now the third most popular activity after internet searches and e-mail (Timimi 2012). However, it has led to concerns about the quality of the information, the ability of lay people to understand it (Gerber/Eiser 2001) as well as potential cyberchondria (Starcevic/Berle 2013). In light of these conflicting perspectives, this paper examines one such source of online information, namely, the patient forum where patients communicate with other patients about a particular medical condition. Although doctor-patient communication in the clinical situation has been extensively researched, little is known about how patient-patient communication is managed in online situations such as patient forums. The purpose of this paper is to contribute to research in that relatively un-researched area by examining how patients manage relational and informational aspects of communication in online patient forums. Whilst a typical interactional structure of the patient forum exchange is question and answer, we focus on responses to questions on patient forums.

This paper reports on the findings of a thematic analysis (Braun/Clarke 2006) of an online thyroid disease patient forum, investigating how interpersonal aspects are negotiated where patients share condition-related knowledge. We identify themes that relate both to informational and relational aspects as well as themes that fit under a new category which we call 'info-relational' as it subsumes informational and relational elements. We discuss a number of theoretical implications, which are valuable as existing health communication models and understandings of patient expertise have yet to catch up with the effects of new media such as online patient forums.
\end{abstract}

\section{Introduction}

The advent of the internet has revolutionised many aspects of health communication. Traditionally, biomedical knowledge was the property and domain of healthcare professionals, and patients thus needed to access this knowledge through their doctor (Heidelberger et al. 2011: 1). However, the advent of online health information has resulted in a plethora of alternative sources of information, democratising access to biomedical knowledge. Patients value and seek online health information (Heidelberger et al. 2011: 2, Mittal et al. 2010: 702, Starcevic/Berle 2013): looking for health care information is now the third most popular online activity after internet searches and e-mail (Timimi 2012). The role of the doctor has correspondingly shifted from that of "sole custodian of medical data" (Eysenbach 2008: np) to being one of many possible sources of biomedical information and advice.

This paper chooses as its object of inquiry the online patient forum where patients communicate online with other patients about a particular health condition. The main purpose of patient forums is to provide a medium where patients with similar concerns can support each other. Forum membership is voluntary, and members can participate to the degree that they wish (Finfgeld 2000 ) either actively or passively, that is without posting, also referred to as 'lurking' (Ziebland/ Wyke 2012: 221). The patient forum is interesting to explore as it is an instance of a health com-

\footnotetext{
* Antoinette Mary Fage-Butler \& Matilde Nisbeth Jensen Department of Business Communication School of Business and Social Sciences Aarhus University Jens Chr. Skous Vej 4 DK-8000 Aarhus $C$ fage-butler@asb.dk-matnj@asb.dk
} 
munication medium where posters are not only patients, dealing with the impact of a condition on their lives, but they also take on a role that was once the sole provenance of the doctor (Orgad 2005: 150): on patient forums, patients seek and receive information, advice and support on their condition from other patients who have the same condition. Some patient forums have doctors as moderators; the patient forum analysed in this paper does not, as we are interested in unmoderated patient-patient communication to explore how patients communicate about their condition without a doctor present.

Not so surprisingly, perhaps, medical professionals have expressed a number of concerns about online patient forums. These include disquiet about the biomedical accuracy of the information that is offered online on patient forums (Deshpande/Jadad 2009, Lewis 2006) though this is not necessarily an issue: a study (Esquivel et al. 2006) found that only 10 of 4600 postings on an unmoderated internet cancer support group were false or misleading, and 7 of these false or misleading statements were corrected within an average of four hours and 33 minutes, suggesting that patients were capable of moderating sites. Another concern has been the likelihood that patient forums propagate cyberchondria, hypochondria brought on by an imbalanced, unqualified reading of online biomedical information (Starcevic/Berle 2013, White/Horvitz 2009).

Despite such concerns, research reveals that patients greatly appreciate online patient forums. Amongst other things, they welcome the sense of safety that derives from the anonymity of online communication (Lindsay et al. 2009: 647). This has been characterised as a "patient-driven relaxation of privacy" (Swan 2009: 513), as patients decide themselves what personal medical information they want to share. Patients have characterised online forums as the ideal medium to communicate without fear of boring, annoying or worrying people in their offline surroundings (van Uden-Kraan et al. 2008: 413).

Besides online forums' value as sites where the degree of personal revelation can be managed, research has identified two other aspects of online patient forums as being particularly beneficial to patients qua patients, namely, their capacity to meet patients' needs for information and support. Heidelberger et al. (2011: 1), for example, point out that an online health network supports knowledge-sharing and provides emotional support. Online forums have been found to help patients manage the extensive amount of health-related information available online by sharing information on how to navigate and interpret the wide array of sources (Swan 2009: 513). Moreover, online patient forums are described as spaces where solidarity and positive regard are shown (Morrow 2006). Wood and Smith (2001, quoted in Morrow 2006) find that patients' engagement in online patient-patient communication can be motivated by their need to communicate with others who are experiencing similar problems.

We take our starting point in previous research which has identified online patient forums as meeting patients' informational and relational needs, as just outlined. However, this article pursues the less examined aspect of how information and support are provided in response to queries posted on online forums. In other words, our interest in this paper lies not so much in what patients feel they receive from patient forums, which is best uncovered by speaking to patients themselves, but in exploring how informational and relational aspects of health communication are actually provided in online patient forum communication, not only in the absence of the doctor but also in lieu of the doctor. Our focus is correspondingly on the responses to posted questions (rather than on the questions), where patients, not doctors, provide responses to posted queries. As Orgad (2005: 150-151) rightly observes, patients' online spaces can be considered "online spaces for reversal: from lay into expert". What is it, then, that happens when patients in the patient forum setting respond to these queries, and in that way, adopt the role once considered exclusively the doctor's?

The data we use to attend to our research purpose are drawn from an online patient forum that is dedicated to patients with thyroid problems. Thematic analysis (Braun/Clarke 2006) is employed to identify how patients manage informational and relational aspects in response to online queries. This division of communicative labour into informational and relational aspects that has 
characterised discussions of the merits of online patient forums, outlined above, has also been identified by Step et al. (2009) and Siminoff/Step (2011) as characterising health communication in the traditional clinical encounter. This classification of health communication into informational and relational sub-components was therefore employed as an initial means of structuring the analysis. The aim of the present study is to improve understandings of how patient-patient communication takes place online, especially given patients' broad enthusiasm for this medium. On the basis of the findings, implications for current understandings of the laity of patients and for theoretical aspects of health communication are discussed.

\section{Data}

An online patient forum for thyroid patients was selected for analysis for two main reasons. First of all, thyroid dysfunction often remains undiagnosed even when patients complain of thyroidrelated symptoms (Landers 2004): it is estimated that almost half of those with thyroid dysfunction are not properly diagnosed (Landers 2004). The thyroid patient advocacy group, Thyroid Patient Advocacy, estimates that 1 in 4 people worldwide have some form of thyroid disease, but that more than half of these do not receive a diagnosis for their condition and are therefore not prescribed appropriate treatment (Thyroid Patient Advocacy 2013). Given the under-diagnosis of thyroid conditions, we anticipated that the problem of missed diagnosis would motivate participation in online thyroid forums. Patient communication about biomedical issues such as (missed) diagnosis was very relevant for us to investigate, particularly given the absence of doctors as moderators on the online forum we were investigating.

The second reason for choosing an online forum for thyroid patients was that the symptoms of thyroid disease are many and diverse, including reduced appetite, feeling cold, thin hair, fatigue, constipation, weight increase, slow reflexes and low heart rate for underactive thyroid (hypothyroidism) (Thyreoidea Landsforeningen 2013); and restlessness, nervousness, shaking hands, fatigue, sweating, muscle fatigue, heart palpitations and weight loss, loose and more frequent stools for overactive thyroid (hyperthyroidism) (Thyreoidea Landsforeningen 2013). Because of the complex nature of the symptoms, thyroid dysfunction can sometimes be misdiagnosed as a psychiatric disease such as stress or depression (Landers 2004). Given the diffuse symptoms associated with thyroid conditions, we anticipated that patients would share their experiential knowledge of the condition online so as to help others achieve a correct diagnosis. Moreover, it was anticipated, given the findings of previous studies of online forums (e.g. Heidelberger et al. 2011, Morrow 2006, Swan 2009), that patients would share knowledge (informational aspects) and support (relational aspects) on how to manage these diverse symptoms which can be very debilitating and frustrating. It is all the more relevant to examine this aspect, as patients' knowledge has often been characterised as 'experiential' and as representing an alternative kind of knowledge to biomedical knowledge (Caron-Flinterman et al. 2005).

An online thyroid forum was identified through the Google search engine using the key words 'thyroid' and 'patient forum', and an active forum was chosen. The forum is publicly available: membership is not required to view forum threads. The number of members registered at the time of writing this paper was just over 1300. For our data, we chose a part of the forum that was dedicated to a general discussion of thyroid issues, and chose all the threads from one week in March 2013 as our data set.

Ethical aspects of researching online patient forums have been discussed by others (e.g. Attard/ Coulson 2012). The data used here were publicly available, and we did not participate actively, but just observed the forum. To protect the identity of the forum participants, we have omitted their online names, kept the quotations included in our analysis as short as possible and sometimes changed quotes slightly (either by substituting words, making clear where any alterations were made by using square brackets, or by adding ' $\mathrm{XX}$ ' where words were removed). 


\section{Method}

To investigate the informational and relational aspects of the patient forum, thematic analysis was employed, as this method enables the identification and analysis of themes in texts. It is much utilised in qualitative research (Boyatzis 1998 in Braun/Clarke 2006: 77, Bryman 2008: 554). As we were interested in characterising the content of forum exchanges, particularly the nature of the responses to questions that are posted online, thematic analysis was ideal as it supports the identification of semantic aspects of texts (Braun/Clarke 2006: 84), or what texts are about. Significantly too, the strengths of thematic analysis in the context of online patient communication have been tried and tested: see, for example, Van Hoof et al. (2013), Attard/Coulson (2012) and Watson et al. (2006), who have all employed thematic analysis as the methodology with which to analyse online patient forums.

We followed Braun/Clarke's (2006) approach to identifying themes, where a theme "captures something important about the data in relation to the research question, and represents some level of patterned response or meaning within the data set" (Braun/Clarke 2006: 82). Drawing on the method described in Braun/Clarke (2006), the process of identifying themes involved the following stages: both of the present authors read through the data separately to become familiar with them before initial codes were generated separately and then agreed upon. As we looked for themes that related to informational and relational aspects of patient communication and therefore operated with some concepts in mind before we started (Braun 2006: 83), the thematic analysis that we conducted was primarily theoretical and deductive as opposed to inductive. However, it was also open to identifying new categories, where the data and existing theory about informational and relational communication (Step et al. 2009, Siminoff/Step 2011) were not aligned, as our Results section will illustrate. Based on our initial codes, all the data went through a full coding by both authors, where new codes were added and the initial codes were refined. The codes were then compared, and themes were identified and double-checked against the data. Finally, passages were selected from the data to illustrate the themes.

Themes are often are expressed as nouns, but it was necessary to include verbs in the theme descriptors as respondents did something in the communicative situation. Including verbs in the descriptors is in keeping with the definition of a theme as something that identifies something salient in the data with respect to the research question (Braun/Clarke 2006: 82). The presence of verbs (gerunds) reflects that we were interested in how informational and relational aspects were negotiated in the threads. See also Attard/Coulson (2012) for a similar approach.

\section{Results}

As mentioned in the Introduction, the primary aim of this paper is to conduct a thematic analysis of informational and relational aspects of patient-patient communication to identify salient themes in our data and thereby shed light on aspects of patient-patient communication in online forums. Our analysis shows that participants' responses to question threads involve many aspects that correspond to both informational and relational communication with themes and sub-themes apparent under both of these categories. However, we also found that we needed to introduce a new category called 'info-relational' where themes related equally to the two categories of 'informational' and 'relational'. In the analysis that follows, the expression 'original poster' is used to refer to the participant who sent out the query and 'respondent' to refer to those individuals who responded to the queries.

\subsection{Informational}

Previous conceptions of informational aspects of health communication (e.g. General Medical Council 2013, Step et al. 2009) understand by 'informational' the expertise a medical practitioner needs to possess to be able to provide relevant and accurate assessment and advice based on bio- 
medical aspects of a condition. This includes biomedical knowledge of symptoms and etiology, and knowledge that supports diagnosis, treatment and prevention.

Biomedicine is present as a recurrent and overarching theme under the category 'Informational' in the data we analysed. The following three sub-themes that relate to the theme of biomedicine were found: providing biomedical information, asking follow-up questions and giving treatment advice. It is useful to point out here that it is not our purpose to comment on the accuracy of the biomedical information provided. Alongside the theme of biomedicine, another related theme is evident in the data: semi-expertise, which is evident in two sub-themes; these are acknowledging lay status and promoting semi-expertise. See Figure 1 for an overview of themes and subthemes that relate to the category 'Informational':

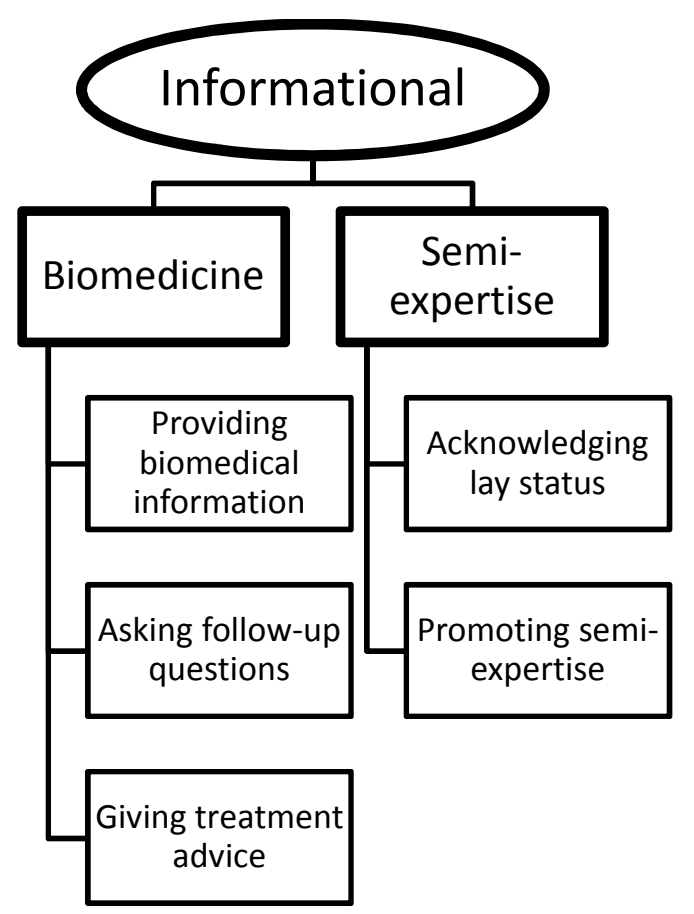

Figure 1. Thematic analysis of data relating to the category 'Informational' showing themes and sub-themes

\subsubsection{Biomedicine}

Thematic analysis revealed that biomedicine is a central theme in the online forum. It is expressed through three recurrent sub-themes.

\section{Providing biomedical information}

Providing biomedical information is a prominent sub-theme under the main theme of biomedicine. Some forum respondents demonstrate considerable biomedical knowledge, which they share in response to the original posters' concerns and questions:

With a swing of 2 in your TSH, your other numbers have also moved and since you feel [unwell] you need to begin having the Free's run.

As illustrated in this example, biomedical knowledge is evident in unexplained biomedical terminology such as 'frees' and the use of the acronym 'TSH'. Biomedical acronyms are widespread in the data set; they include 'ANA', 'TPO', 'TT', 'TIBC', 'FNA'. Forum respondents draw extensively on biomedical knowledge about the management of thyroid-related conditions: 
I bet if your FT-4 went up a bit your TSH would drop.

Persons w/thyroid disease often have low ferritin. I consider this to be yet another symptom (indicator).

Respondents are also equipped with biomedical knowledge about issues that are not directly related to thyroid disease:

The fat-soluble vitamins are A, D, E and K. These CAN become deficient when we have malabsorption problems or any kind of increased GI motility.

Vitamin D deficiency causes decreased calcium absorption from your gut, so your body ends up having to break down bone to keep your serum calcium normal. This could account for your back pain.

\section{Asking follow-up questions}

When respondents need more information from the original poster, follow-up questions are often asked such as:

Would you be able to provide a list of ALL the meds you are taking? Might be helpful!

Okay, so your TSH is super-low, but what about your Free T4 and Free T3?

The function of these follow-up questions seems to be to ensure that correct and best advice is given. These questions have their counterparts in the kind of communication that takes place in doctors' surgeries (Ely et al. 2000).

\section{Giving treatment advice}

Forum respondents also offer advice to original posters on tests to be undertaken and biomedical treatments to be followed:

It might be wise to get an ultra-sound of your heart. You may have the gushing sound from mitral prolapse.

Have you had your blood pressure checked recently? If not then I advise you to do so, there is a real risk of strokes/heart disease when hyper.

Their recommendations for treatments and courses of action are based on biomedically-underpinned understandings:

If you are hyper - give anti thyroid meds 18 months to put you in remission.

With eye involvement your best bet really is the surgery XX although it's said that a round of glucocorticoids before during and after RAI can lessen or even prevent further eye problems.

Summing up, thematic analysis of data that relate to the theme of biomedicine reveals that biomedical information is readily provided in response to original posters' queries and concerns. Sometimes follow-up questions are used to ensure that the respondent has a comprehensive picture before advice is offered to the original poster. Recommendations for biomedical treatments or courses of action are also suggested, and patients are encouraged to learn more about their condition. The cumulative effect is threads that are saturated with biomedical information and advice: biomedicine constitutes a very salient theme in this online forum, expressed in complex and rather subtle ways.

\subsubsection{Semi-expertise}

At the same time as biomedical information is a significant theme in the data, another theme, which is related but contrasting and provides counterpoint to the theme of biomedical information, is the theme of semi-expertise, where semi-expertise relates to the status of the posters 
as semi-experts. Under semi-expertise, two sub-themes are apparent. These reflect respondents' awareness of the limits of their own biomedical knowledge, on the one hand, and promotion of the semi-expertise of the original posters, on the other hand. These sub-themes we have therefore called 'Acknowledging lay status' and 'Promoting semi-expertise'.

\section{Acknowledging lay status}

Respondents' acknowledgement of their own semi-expertise is evident in the fact that many have disclaimers under their profiles that show up when they post which underline that the advice given should not be deemed to be expert and recommend that posters see a qualified doctor before acting on any of the information on the forum:

No doctor degrees here.

The standard disclaimer included at the end of many of the postings that acknowledge the lay status of the posters is the following:

Please see a qualified doctor before [using and relying on] any information found here.

Respondents' awareness of gaps in their biomedical knowledge is also explicitly stated in their responses to threads:

I think it really varies between individuals but I'm not well educated in this area.

I personally am not aware of any large-scale studies XX, but that doesn't mean they haven't been done.

Respondents' acknowledgement of their lay status is also evident when they refer to approved sources of biomedical information such as their doctor, existing literature or medical webpages:

My surgeon's guiding principle is XX.

I just looked up symptoms of persistent, low ferritin levels and they include: XX.

According to the Mayo Clinic: "Many people with no disease have positive ANA tests XX".

\section{Promoting semi-expertise}

Another sub-theme that relates to the theme of semi-expertise has to do with respondents' promotion of the original posters' knowledge of their condition and how to treat it, so that they too can become more expert in understanding thyroid disease and its treatment. This sub-theme is evident in respondents' provision of web links (sometimes to articles in academic journals), and their encouragement of the original posters to read these:

\footnotetext{
Here is some info on Hashimoto's which may be helpful: XX

http://emedicine.medscape.com/article/120937-diagnosis.

Just in case you haven't done research on this yet, here are a few links:

http://www.ncbi.nlm.nih.gov/pmc/articles/PMC1584230/.

http://www.psycheducation.org/depres...thiumRisks.htm.
}

These posts reveal an assumption that original posters' lay status can be addressed: it is possible to acquire biomedical understanding of one's condition, and in this way progress from being lay or knowing very little about the condition and how to manage it towards having a degree of semiexpertise.

Summing up the findings under the category of 'Informational', whilst the theme of biomedicine relates to respondents adopting a role similar to that of the doctor-providing biomedical information, asking additional questions and suggesting treatments - the theme of semi-expertise 
provides a nuancing of this, where gaps in the knowledge of the respondents and original posters are recognised. The knowledge-status of respondents thus hovers between lay and expert, and is considered modifiable as it can become more expert (or less lay) when patients proactively equip themselves with biomedical information.

\subsection{Relational}

Step et al. (2009) and General Medical Council (2013) define 'relational' aspects of clinical communication as having to do with doctors' possession of the people or communication skills to manage the often challenging interpersonal aspects that can be associated with the clinical encounter with the patient. Here, we examine patients' possession and communication of such relationality, another way in which this study is noteworthy. Two main themes were evident in the data that related to different kinds of support-giving and acknowledgement (See Figure 2):

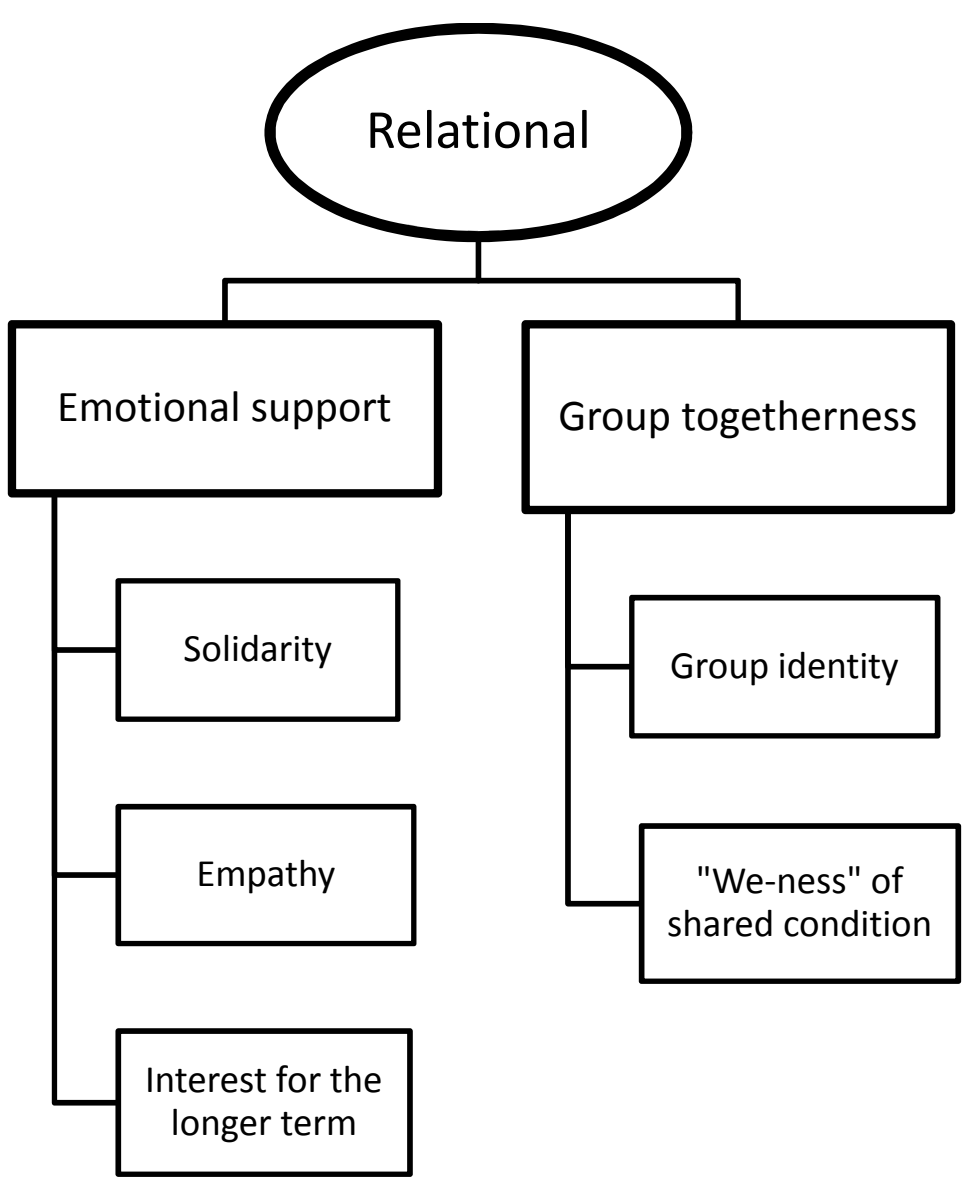

Figure 2. Thematic analysis of data relating to the category 'Relational'

\subsubsection{Emotional support}

Emotional support is a prominent theme in the data. This is particularly evident in postings that reflect respondents' awareness of the emotional needs of the original poster.

\section{Solidarity}

In the following examples, support is offered through expressions of solidarity that underline the commonality of thyroid patients' experiences: 
Good luck, and don't get discouraged. I am currently going through similar. It seems to take some time to get a diagnosis.

Let me know any XX symptoms you're having, and I would be happy to help you come up with a list of things to ask your doctor.

I hope you don't have XX, but if you do, [it can be managed]. Most of the time I am not even aware of the fact that I have XX.

Such expressions indicate a community of interests and can help to engender group loyalty. This is particularly important given the problematic aspects of diagnosing, treating and living with thyroid conditions.

\section{Empathy}

At times, thematic analysis reveals empathy, where the respondent reflects his/her ability to imagine the emotional state of the other, particularly in relation to anxieties about results and treatments:

I wouldn't read too much into the elapsed time.

You will be SO relieved when this is behind you.

By resonating emotionally and expressing affinity with the original poster, the respondent recognises and validates emotional aspects of the original poster's experiences of the thyroid condition and its treatment.

\section{Interest for the longer term}

Another way in which emotional support is evident is in statements of well-wishing, indicating a benevolent attitude to the anonymous other:

Prayers for a fast recovery after surgery! Will be thinking of you on the $8^{\text {th }}$ for sure!

I wish you the best of luck and hope they figure things out for you soon!

Similarly, statements are also included in postings that indicate interest in the longer term welfare of the original poster:

When you feel up to it, let us know how you are doing.

Keeping you in my thoughts and please let us know what you decide to do.

Please do let us know about the EBV.

Such statements suggest genuine concern for the well-being of the original poster, indicating that a relationship which includes a sense of commitment has been struck up. This seemingly genuine concern for strangers that post on the thyroid patient forum is also evident in posts where respondents reply to a thread, despite not being able to provide any practical help or advice:

I think it really varies between individuals but I'm not well educated in this area. I've never actually had a XX in 15 years of being hypo so I haven't read much about it.

\subsubsection{Constructing group togetherness}

Another theme that has to do with relational aspects of the patient forum communication is constructing group togetherness. This is evident in two sub-themes which we call 'group identity' and "we-ness" of shared condition'. 


\section{Group identity}

The thyroid patient forum members are described as constituting a group. This is particularly evident when new posters are welcomed, sometimes multimodally through icons, to the group:

We rock!

Welcome, sorry for what brings you here.

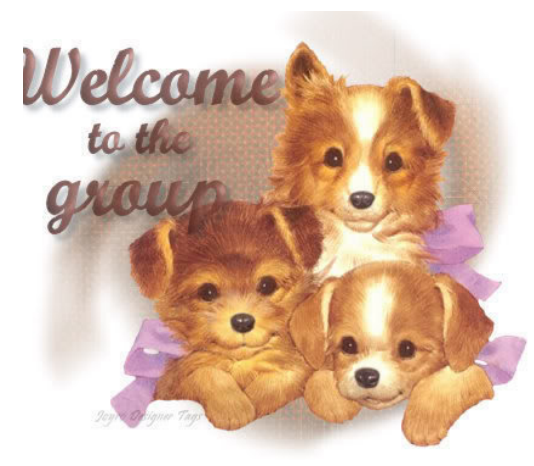

\section{“We-ness" of shared condition}

The togetherness of the group relates primarily to the fact that they share a biological condition and that their lived experience of that condition inevitably results in some commonality:

I also don't expect a solution soon. Since thyroid hormone replacement is so readily available, relatively inexpensive, and usually well-tolerated, it's hard to imagine us thyroid folks will be at the top of the autoimmune queue.

A lot of us here have more than one autoimmune disease going on. It seems to be part and parcel. Sad, but true.

Hang in there! It is comforting to know others are in the same boat.:)

The relational category thus spans kindness and concern for fellow thyroid sufferers as well as emphasising the 'we-ness' of the group through statements that relate to shared experiences and a common identity. Most striking of all, perhaps, are the seemingly genuine expressions of empathy and concern for other posters, particularly given the anonymity of the medium. This is reminiscent of the ethical perspectives of Levinas (1996) which emphasise people's fundamental and unavoidable responsibility for the 'other'.

\subsection{Info-relational}

As stated at the beginning of the Results section, the category 'Info-relational' was coined in response to the findings of our thematic analysis of the online patient forum. Our thematic analysis suggests that the dichotomous categories of informational and relational, which characterise previous health communication models for the clinical situation and which also have been used to describe the function of patient forums, appear to be in need of review. The data we analysed pointed to the existence of a third category, which we call 'Info-relational'. It is used for themes that we found fitted equally well under the informational and relational categories. Under the category 'Info-relational', two themes were identified: sharing experiences and knowledge and endorsing one another's knowledge. See Figure 3: 


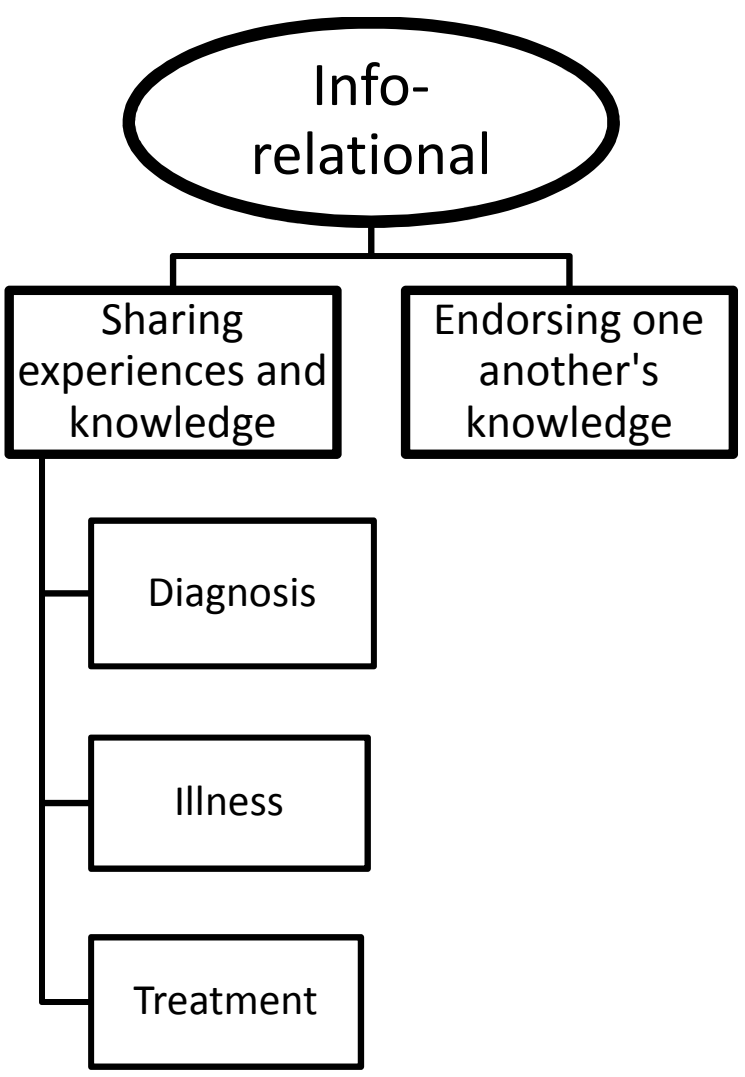

Figure 3. Thematic analysis of data relating to the category 'Info-relational'

\subsubsection{Sharing experiences and knowledge}

Sharing experiences and knowledge spans two kinds of knowledge that patients possess: (1) personalised biomedical knowledge, where biomedical knowledge, institutions and practices are transformed through patients' experiences that filter these aspects of biomedicine through patients' individual subjectivities, and (2) the nature of patients' experiential knowledge which lies outside of the professional domain of biomedicine (Caron-Flinterman et al. 2005) and is underpinned by a very different epistemology. Largely due to the personal embeddedness of the knowledge of both of these aspects, the theme of 'sharing experiences and knowledge' spans relational and informational categories. The theme of 'sharing experiences and knowledge' was expressed thematically in the ways in which respondents shared their personal experiences about diagnosis, illness and treatment.

\section{Diagnosis}

Many of the threads referring to experiences of biomedicine reflect the difficulties experienced by the participants in getting a proper diagnosis. Respondents share their experiences often through short narratives:

I had a tough XX getting a diagnosis XX I kept hearing menopause, ugh.

NOT ONE BLOOD TEST FOR XX IS ABNORMAL over the last 20+ years and yet I have the disease XX Just because it doesn't show up in the blood doesn't mean you don't have the disease.

Narratives that relate to difficulties at the diagnosis stage often are accompanied by criticism of the biomedical model and doctors: 
The funny thing is I asked them if XX makes a difference for cancer recurrence and they don't even know if it does, so basically they are keeping me hyper based on a theory and not scientific proof!

Another post ends with the comment "I don't think probably is a good enough answer; do you?" referring to the original poster's comment that their doctor had said that s/he probably has autoimmune hypothyroidism:

Clearly this doctor is not interested in pursuing this any further so I hope you can find one that will run these tests and also order an ultra-sound of the thyroid.

Here, patients' personally invested interests in their own well-being are contrasted with mechanistic aspects of biomedicine.

\title{
Illness
}

Respondents often share quite detailed information about their experience with their illness, including diagnosis, medication, dose, surgery and their well-being in relation to these:

\begin{abstract}
I was on XXmg of methimazole when I was first diagnosed with Graves...I was extremely hyper. I also took propanolol for the rapid heart rate. After XX years, I was still on about XXmgs a day up until the day I had a total thyroidectomy 3 months ago. ... Methimazole is help but not a cure. I'm not $100 \%$ regulated on replacement meds yet and some days are bad but for the most part I feel great compared to the 2 years of hyper hell.
\end{abstract}

In the following extract, the respondent replies to a thread from an original poster regarding frustrations about being unable to sleep due to hearing blood circulating in one ear. The respondent chooses to explain her/his experience of the illness including medical history and lab values before commonality is established, where an identical problem is explained:

\footnotetext{
I have been majorly hyper since July XX only just been diagnosed with Graves. My TSH when first diagnosed was XX so non existent, and blood pressure XX, I do remember before being medicated that I also could hear my heart in my ears mainly at night when trying (Trying very hard) to sleep a kind of swooshing sound...
}

This quotation shows that experiential knowledge, which is personal and relational, is mixed with the sharing of biomedical knowledge, which is an "informational" aspect (Step et al. 2009, Siminoff/Step 2011), such as the naming of medications, blood test values and symptoms.

\section{Treatment}

Participants argue for certain choices of treatment and share how this choice is often based on what they find is best for them as individuals, in this way emphasising that for health issues, one size does not fit all.

I opted for the surgery ... for me anyway, XX have their own inherent problems.

Very specific information is given on managing different treatment regimes, which is sometimes rooted in personal experience and the experiences of others:

\footnotetext{
Lots of people iced their neck...I didn't because having something touch my neck [freaked] me out, but you might want to prepare. I read on pinterest that the best "homemade" ice packs are actually dish washing soap (liquid) frozen in zip lock freezer bags.
}

My saviors were 1) v-necks/button up/zip up shirts (I lived in my husband's v-neck undershirts for weeks ()), and 2) insulated cups with straws. I couldn't [handle] drinking anything hot, but ice cold drinks were great. I also had some minor issues with swallowing - I just had to take little sips or small bites \& chew well and then swallow carefully. For some reason, having a straw really, really helped.

XX's advice above is good. Plan to get plenty of rest. I didn't ice, and I did fine. 
Such advice not only contains information about personal experiences, but it often communicates empathy regarding the difficulties of managing the treatment:

RAI scared me too. I had already had XX the thyroid removed years ago and decided the surgery was a better fit for me. I have minimal lid lag on my left eye as well as it being photophobic. I was too far gone (a mental and physical wreck) to even begin to think of remission, so I [had] surgery as quickly as possible. I don't regret it. In fact, I am really glad I had it. It took a little while to get the replacement at a correct dose. In terms of the Graves and thyroid issues, I have recovered really well.

\subsubsection{Endorsing one another's knowledge}

Info-relational communication is not only evident towards the original posters, but also laterally towards other respondents who have supported the original posters with advice and information:

XX's advice above is good. Plan to get plenty of rest. I didn't ice and I did fine.

To be honest, you got the best info and opinion you could possibly get from XX.

Let's see what others have to say.

But XX is right: the ANA positive result can mean a number of different things, which means more testing is necessary to rule out $\mathrm{XX}$.

Positive references to the quality of the information and advice given by other posters add to group cohesion, a relational issue.

\section{Discussion}

\subsection{The nature of the informational aspects}

A significant finding of the analysis is that respondents often possess considerable biomedical knowledge, which is acquired from sources such as doctors, other patients and journal articles, and which is evident in the way in which they use very specialised terminology and acronyms. Biomedical information is of particular value to newcomers to the group who seek advice on how to navigate all aspects of medical care, particularly where the posters' doctors are characterised as having a gatekeeping function. Not only is biomedical information provided which may support the original posters, but we also found several examples where respondents adopt a role similar to that of the doctor in the clinical situation: they ask clarifying questions, request further information and suggest treatment. In doing so, they appear to abandon the traditional role of patient and adopt that of medical practitioner. Such statements are, however, often modified by the use of disclaimers which underline the respondents' lay status.

Sharing biomedical knowledge helps to democratise expert knowledge, and it may also have positive consequences for the quality of treatment patients receive. Following Cicourel (1999), Sarangi argues that "some patients may be better historians of their illnesses and so their rich and accurate accounts of symptoms can make a difference to the quality of health care delivery" (2001: 5). Moreover, once empowered, patients are in a position to engage in more egalitarian relationships with their health professionals (Roberts 1999: 87). The informational aspect of patient forums can clearly contribute to this kind of health competence: it can be educational and empowering. Patient empowerment which operates horizontally between patients is different to patient empowerment as it has been defined for the clinical situation (Feste/Anderson 1995, Holmström/ Röing 2010). According to Roberts (1999), patient empowerment in the clinical situation entails a redistribution of power between patients and physicians, with health professionals empowering patients (Roberts 1999: 85), leading to suspicions that patient empowerment is disguised authoritarianism (Skelton 1994). Our data tell a different story - it is patients that empower patients, without the involvement of the doctor. In that vein, we agree with Bos et al. (2008), who have argued that true patient empowerment becomes feasible with recent developments in the appli- 
cation of the Internet, especially the potential of Web 2.0, hence the evolution of the concept Patient 2.0 Empowerment. Similarly, van Uden-Kraan et al. (2008: 414) suggest that participation in online support groups seems to offer the patient assistance with what is demanded of the new empowered patient.

\subsection{The nature of the relational aspects}

Relational communication themes reflected the importance of group togetherness communicated through a 'we are in the same boat' discourse and a benevolent attitude to the anonymous other, which includes well-wishing and empathy, with emotional support offered for the longer term. Relationship-building has been found to be integral to the patient forum: van Uden-Kraan et al. (2008), for example, conclude that individual empowerment in the context of online support groups is "linked with group and community empowerment in a dialectical relationship" (2008: 415). This finding supports our results that empowerment which is experienced at an individual level is often the product of relational group interaction.

Relational aspects of online patient forum communication share with patient-centred communication (Balint 1969, Mead/Bower 2000) the view that the original patient poster is not only a patient, but also a person who has unique needs. Indeed, whilst online forum communication necessarily means that participants are not physically and temporally co-present, it is possible that the asynchronous and anonymous quality of the online forum supports greater openness and support. This is, indeed, indicated by Kummervold et al.'s (2002) study where 75\% of patients state that they preferred online forums to face-to-face settings when sharing their mental health problems, because their identities were concealed through the use of pseudonyms. The anonymity of the medium may paradoxically support a greater degree of personal revelation and investment in promoting others' wellbeing, though there needs to be greater understanding of the nature of this support, and how it compares with the support that is offered when both parties are physically present and anonymity is not an option.

\subsection{The nature of the info-relational aspects}

In our analysis, we found that informational and relational aspects are often intertwined. This is evident, for example, when respondents share their experiences of their illness and its treatment, including aspects relating to diagnosis, medication, dosage and surgery, where there is also emphasis on supporting the original poster, given the commonality of the illness that the original poster and the respondent share. Our findings thus support Shakespeare (2013), who argues that even in the clinical setting, "technical versus caring skills is a false dichotomy". Our data also reveal that in the online thyroid patient forum, the neat dichotomy between informational and relational communication that has characterised health communication models and has even been used to describe what patients receive from online patient forums needs to be reviewed (e.g. Heidelberger et al. 2011).

There are two main ways in which info-relational aspects are evident in the online thyroid patient forum. First of all, patients share experiences of their encounters with the biomedical system. Short narratives are often used to communicate these experiences. Information about the system is thereby filtered through personal experience which is intended to support the original posters in navigating the system. Given this focus, it is also under this theme, particularly in relation to diagnosis, that criticism of both the biomedical model and doctors is apparent.

Secondly, there is the issue of the unique expertise of patients - their experiential expertise. Experiential expertise has been defined as knowledge that is derived from sensory reactions, such as experiential dimensions of symptoms and physical reactions to illness (Caron-Flinterman et al. 2005), meaning that "people have their own embodied experience to draw on when deciding whether and how to act" (Ziebland/Wyke 2012: 220). Patients on the online thyroid patient forum certainly share such experiential knowledge. Indeed, we found evidence that the thyroid forum 
patients offer information that according to Ferguson "some clinicians may consider secondary e.g., practical coping tips and the psychological and social aspects of living with the condition" (Ferguson 1999: np). An example from our data is the sharing of what type of shirt to wear postsurgery and how best to ice. Thus, the patient forum facilitates the sharing of experiential knowledge, a function which is not fulfilled in clinical encounters where doctors lack the knowledge that is derived from having and experiencing the condition concerned. The rich 'insider' information associated with experiential knowledge may help to explain the popularity of online patient forums.

Info-relational communication in the forum also potentially promotes patient empowerment: by sharing experiential knowledge, forum participants have a more informed ground on which to base their decisions, coupled with the relational dimension which might encourage them to act on their symptoms. An example of this kind of encouragement is the quotation: "I don't think probably is a good enough answer; do you?" Our results support the findings of other studies of online media which have shown that the main empowering processes that occur within the support groups were the exchange of knowledge and the sharing of experiences (van Uden-Kraan et al. 2008: 406).

The endorsement of other forum participants' knowledge is another theme that is included in this section as it exists at the intersection between informational and relational aspects. Much like the use of references in academic writing (Hyland 2004), it functions to validate knowledge at the same time as having a socially cohesive function.

\section{Conclusion}

Whilst previous research has examined how and whether online forums satisfy patients' needs (Attard/Coulson 2012, Mo/Coulson 2010, Ziebland/Wyke 2012), our paper differs in two main ways: we investigate how support is operationalised communicatively, and our analysis, as it is textual, focuses on what forum respondents give original posters, not necessarily what the original posters need.

Empirically, this paper sheds important light on how patients communicate with other patients in online situations. It illustrates how patient-patient communication has striking similarities with aspects of doctor-patient communication as it includes the sharing of biomedical knowledge on diagnosis, managing illness and treatment. However, whilst supporting patients' empowerment by providing extra information is also an aim of communication in the clinical situation (e.g. Little et al. 2004), information-provision in the thyroid forum is often angled more sharply against the system, particularly when it relates to empowering patients with information so that missed diagnoses can be rectified and appropriate tests conducted. Moreover, patient-patient communication clearly comprises aspects that cannot be met in traditional doctor-patient communication as it incorporates experiential knowledge, empathetic support drawing from common experience and 'we-ness' or group solidarity.

Theoretically, this paper is significant as it provides evidence that the simple dichotomy into relational and informational aspects that underpins many understandings of health communication for the clinical situation does not apply to online patient forums. Quite a few themes spanned the relational and informational categories, leading to our development of a new category which we called 'info-relational'. As this category is new, further studies need to investigate the nature and extent of info-relationality in computer-mediated patient-patient communication.

The paper has its limitations: thematic analysis is interpretative, but we have aimed to enhance its rigour by both authors analysing the data independently, as described in the Methods section. Also, limitations in the data point in the direction of future studies. The data set was taken from one thyroid online forum, so it is not possible to derive generalisations that are sure to apply to other conditions. It is also possible that the week we chose was not representative of the postings 
on the forum as a whole. Further research should therefore be conducted to investigate whether the thematic patterns identified in our present data are evident more widely.

Another important follow-up study relates to lay-friendly language. It is often argued that patient information should be kept simple and be expressed in lay language (Sand-Jecklin 2007, Stableford/Mettger 2007). Indeed, van Uden-Kraan et al. (2008), who interviewed patients about their online forum participation, found that patient forums were appreciated because the information was understandable as it was formulated in patients' 'own language' without any 'medic's speak' (van Uden-Kraan et al. 2008: 408). The present study, however, revealed a different picture as the threads include an extensive use of expert thyroid-related terminology and acronyms. The fact that such terminology is used without glossing suggests that thyroid-related acronyms and specialist terms appear not to be considered beyond other patients' grasp, even if they are newcomers to the forum. Not simplifying these terms for other patients may also have been motivated by the will to empower other participants with biomedical terminology so that they could better navigate the biomedical system. Thus, future studies that examine the use of (expert) terminology and language in patient forums could be very valuable.

Given the gaps in empirical and theoretical understandings of communication in online patient forums, there is clearly a need for on-going research in this area. It is our hope that the research conducted here contributes to greater understanding of the implications of the cataclysmic shift that characterises Health 2.0 (Van De Belt et al. 2010), particularly in relation to the nature of patient-patient communication, stimulating further research.

\section{References}

Attard, Angelica/Coulson, Neil S. 2012: A thematic analysis of patient communication in Parkinson's disease online support group discussion forums. In Computers in Human Behavior 28, 500-506.

Balint, Enid 1969: The possibilities of patient-centered medicine. In The Journal of the Royal College of General Practitioners 17, 269-276.

Bos, Lodewijk/Marsh, Andy/Carroll, Denis/Gupta, Sanjeev/Rees, Mike 2008: Patient 2.0 Empowerment. Proceedings of the 2008 International Conference on Semantic Web \& Web Services SWWS08, 164-167.

Braun, Virginia/Clarke, Victoria 2006: Using thematic analysis in psychology. In Qualitative Research in Psychology 3, 77-101.

Bryman, Alan 2008: Social Research Methods. Oxford: Oxford University Press.

Caron-Flinterman, J. Francisca/Broerse, Jacqueline E.W./Bunders, Joske F.G. 2005: The experiential knowledge of patients: a new resource for biomedical research? In Social Science \& Medicine 60, 2575-2584.

Cicourel, Aaron V. 1999: The interaction of cognitive and cultural models in health care delivery. In Sarangi, S./Roberts, C. (eds.), Talk, Work and Institutional Order: Discourse in Medical, Mediation and Management Settings, 183-224.

Deshpande, Amol/Jadad, Alejandro R. 2009: Trying to measure the quality of health information on the internet: is it time to move on. In Journal of Rheumatology 36, 1-3.

Ely, John W./Osheroff, Jerome A./Gorman, Paul N./Ebell, Mark H./Chambliss, M. Lee/Pifer, Eric A./Stavri, P. Zoe 2000: A taxonomy of generic clinical questions: classification study. In British Medical Journal 321, 429-432.

Esquivel, Adol/Meric-Bernstam, Funda/Bernstam, Elmer V. 2006: Accuracy and self correction of information received from an internet breast cancer list: content analysis. In British Medical Journal 332, 939-942.

Eysenbach, Gunther 2008: Medicine 2.0: social networking, collaboration, participation, apomediation, and openness. In Journal of Medical Internet Research 10, np.

Ferguson, Tom. Can Useful and Reliable Online Health Resources be Produced by 'Medically Unqualified' Persons? [online]. http://www.fergusonreport.com/articles/fr079902.htm (accessed 6 June 2013).

Feste, Catherine/Anderson, Robert M. 1995: Empowerment: from philosophy to practice. In Patient Education and Counseling 26, 139-144.

Finfgeld, Deborah L. 2000: Therapeutic groups online: the good, the bad, and the unknown. In Issues in Mental Health Nursing 21, 241-255.

General Medical Council. Good Medical Practice: Apply knowledge and experience to practice [online]. http://www. gmc-uk.org/guidance/good_medical_practice/apply_knowledge.asp (accessed 10 May 2013). 
Gerber, Ben S./Eiser, Arnold R. 2001: The Patient-Physician Relationship in the Internet Age: Future Prospects and the Research Agenda. In Journal of Medical Internet Research 3, e15.

Heidelberger, Cory Allen/El-Gayar, Omar/Sarnikar, Surendra 2011: Online Health Social Networks and Patient Health Decision Behavior: A Research Agenda System Sciences (HICSS), 2011 44th Hawaii International Conference on System Sciences. IEEE, 1-7.

Holmström, Inger/Röing, Marta 2010: The relation between patient-centeredness and patient empowerment: A discussion on concepts. In Patient Education and Counseling 79, 167-172.

Hyland, Ken 2004: Disciplinary Discourses. Social Interactions in Academic Writing. Ann Arbor: University of Michigan Press.

Kummervold, Per E./Gammon, Deede/Bergvik, Svein/Johnsen, Jan-Are K./Hasvold, Toralf/Rosenvinge, Jan H. 2002: Social support in a wired world: use of online mental health forums in Norway. In Nordic Journal of Psychiatry 56, 59-65.

Landers, Susan J. 2004: Overlooked, underdiagnosed? Thyroid disease poses a challenge. American Medical News [online]. http://www.amednews.com/article/20040301/health/303019968/4/.

Levinas, Emmanuel 1996: Is ontology fundamental? In: Perperzak, A. T./Critchley, S./Bernasconi, R. (eds.), Emmanuel Levinas: Basic Philosophical Writings. Bloomington: Indiana University Press, 1-10.

Lewis, Tania 2006: Seeking health information on the internet: lifestyle choice or bad attack of cyberchondria? In $M e$ dia, Culture \& Society 28, 521-539.

Lindsay, Sally/Smith, Simon/Bellaby, Paul/Baker, Rose 2009: The health impact of an online heart disease support group: a comparison of moderated versus unmoderated support. In Health Education Research 24, 646-654.

Little, Paul/Dorward, Martina/Warner, Greg/Moore, Michael/Stephens, Katharine/Senior, Jane/Kendrick, Tony 2004: Randomised controlled trial of effect of leaflets to empower patients in consultations in primary care. In British Medical Journal 328, 441-444.

Mead, Nicola/Bower, P. 2000: Patient-centeredness: A review of the literature. In Patient Education and Counseling 48, 51-61.

Mittal, Mayank K./Dhuper, Sonal/Siva, Chokkalingam/Fresen, John L./Petruc, Marius/Velazquez, Celso R. 2010: Assessment of email communication skills of rheumatology fellows: a pilot study. In Journal of the American Medical Informatics Association 17, 702-706.

Mo, Phoenix K.H./Coulson, Neil S. 2010: Living with HIV/AIDS and use of online support groups. In Journal of Health Psychology 15, 339-350.

Morrow, Phillip R. 2006: Telling about problems and giving advice in an Internet discussion forum: some discourse features. In Discourse Studies 8, 531-548.

Orgad, Shani 2005: Storytelling online: Talking breast cancer on the Internet. New York: Peter Lang.

Roberts, Kathleen Johnston 1999: Patient empowerment in the United States: a critical commentary. In Health Expectations 2, 82-92.

Sand-Jecklin, Kari 2007: The Impact of Medical Terminology on Readability of Patient Education Materials. In Journal of Community Health Nursing 24, 119-129.

Sarangi, Srikant 2001: Editorial: On demarcating the space between 'lay expertise'and 'expert laity'. In Text - Interdisciplinary Journal for the Study of Discourse 21, 3-11.

Shakespeare, Tom 2013: A point of view: How important is compassion in healthcare? BBC News magazine [online]. http://www.bbc.co.uk/news/magazine-22773043.

Siminoff, Laura A./Step, Mary M. 2011: A comprehensive observational coding scheme for analyzing instrumental, affective, and relational communication in health care contexts. In Journal of Health Communication 16, 178-197.

Skelton, Robert 1994: Nursing and empowerment: concepts and strategies. In Journal of Advanced Nursing 19, 415423.

Stableford, Sue/Mettger, Wendy 2007: Plain Language: A Strategic Response to the Health Literacy Challenge. In Journal of Public Health Policy 28, 71-93.

Starcevic, Vladan/Berle, David 2013: Cyberchondria: towards a better understanding of excessive health-related Internet use. In Expert Review of Neurotherapeutics 13, 205-213.

Step, Mary M./Rose, Julia Hannum/Albert, Jeffrey M./Cheruvu, Vinay K./Siminoff, Laura A. 2009: Modeling patientcentered communication: Oncologist relational communication and patient communication involvement in breast cancer adjuvant therapy decision-making. In Patient Education and Counseling 77, 369-378. 
Swan, Melanie 2009: Emerging patient-driven health care models: an examination of health social networks, consumer personalized medicine and quantified self-tracking. In International Journal of Environmental Research and Public Health 6, 492-525.

Thyreoidea Landsforeningen. For højt stofskifte [online]. http://www.thyreoidea.dk/fakta/for-hoejt-stofskifte.html (accessed 9 May 2013).

Thyreoidea Landsforeningen. For lavt stofskifte [online]. http://www.thyreoidea.dk/fakta/for-lavt-stofskifte.html (accessed 9 May 2013).

Thyroid Patient Advocacy. What's it all about? [online]. http://tpauk.com/content/994/thyroid-conditions/ (accessed 9 May 2013).

Timimi, Farris K. 2012: Medicine, morality and health care social media. In BMC Medicine 10, 83.

Van De Belt, Tom H./Engelen, Lucien J.L.P.G./Berben, Sivera A.A./Schoonhoven, Lisette. 2010: Definition of Health 2.0 and Medicine 2.0: A Systematic Review. In Journal of Medical Internet Research 12, e18.

Van Hoof, Wannes/Provoost, Veerle/Pennings, Guido 2013: Reflections of Dutch patients on IVF treatment in Belgium: a qualitative analysis of internet forums. In Human Reproduction 28, 1013-1022.

van Uden-Kraan, Cornelia F./Drossaert, Constance H.C./Taal, Erik/Shaw, Bret R./Seydel, Erwin R./van de Laar, Mart A.F.J. 2008: Empowering processes and outcomes of participation in online support groups for patients with breast cancer, arthritis, or fibromyalgia. In Qualitative Health Research 18, 405-417.

Watson, Mohinder/Peacock, Susi/Jones, Derek 2006: The analysis of interaction in online focus groups. In International Journal of Therapy and Rehabilitation 13, 551-557.

White, Ryen W/Horvitz, Eric 2009: Cyberchondria: studies of the escalation of medical concerns in web search. In ACM Transactions on Information Systems (TOIS) 27, 23.

Ziebland, Sue/Wyke, Sally 2012: Health and illness in a connected world: how might sharing experiences on the internet affect people's health? In Milbank Quarterly 90, 219-249. 